\title{
Active packaging of fish gelatin films with Morinda citrifolia oil
}

\begin{abstract}
Active packaging is of interest in helping to prevent autoxidation process in foods. Morinda citrifolia contains a wide range of antioxidants such as ascorbic acid, terpenoids, and polyphenols. The purpose of this study was to determine the potential of Morinda citrifolia as a natural antioxidant in an active packaging film. Fish gelatin films incorporated with 1-3\% of Morinda citrifolia oil (MO) were used to prepare antioxidant films. It was found that the incorporation of $\mathrm{MO}$ would not affect the thickness and solubility of gelatin films, independent of concentration. However, the solubility ranging from $13.4 \%$ to $13.8 \%$ might be considered low for these films. As for the mechanical properties, Young's modulus and elongation at break were not affected significantly by incorporation of 1-3\% MO ( $>0.05)$. As for the tensile strength, fish gelatin film incorporated with 1-3\% MO showed a higher value than control $(\mathrm{p} \leq 0.05)$. The opacity between the samples and control varied statistically with the highest value with films containing $3 \%$ oil $(\mathrm{p} \leq 0.05)$. However, increasing the MO concentrations would decrease the water vapor permeability ( $p>0.05)$. DPPH (2,2-diphenyl1-picrylhydrazyl) was used to determine the antioxidant activity and the result increased significantly $(\mathrm{p} \leq 0.05)$ from $9 \%$ to $16 \%$ with the increase of oil concentration, suggesting MO incorporation in films as potential means of active packaging.
\end{abstract}

Keyword: $\quad$ Active packaging; Gelatin; Morinda citrifolia; Antioxidant; Noni; Mengkudu 